\title{
Unit Per Gram Hemoglobin
}

National Cancer Institute

\section{Source}

National Cancer Institute. Unit Per Gram Hemoglobin. NCI Thesaurus. Code C105522.

Unit of arbitrary substance concentration (biologic activity concentration) defined as the concentration of one unit per gram of hemoglobin. 